\title{
Simulation of Generator Unit in Thermal Power Plant Using Labview
}

\author{
Shiyam Sundar S, Embedded System \\ Department of Electronic and Communication Engineering, Sathyabama University, Chennai,Tamil Nadu.
}

\begin{abstract}
Laboratory Virtual Instrumentation Engineering Workbench (LabVIEW) are widely used in industry for supervisory control and data acquisition of industrial processes. This paper describes simulation of system for laboratory based thermal power plant generator setup using LabVIEW data logging and supervisory control (DSC) module. Using the input, output and functional parameters the simulation of generator unit and alarm handling technique is achieved.
\end{abstract}

\section{Introduction}

The generator to be simulated using LabVIEW consist of two pole salient type rotor. It is to be simulated with the reference of generator unit in thermal power station at Neyveli Lignite Corporation(NLC), Neyveli. The generator is simulated to produce $210 \mathrm{MW}$ (Mega Watt) operating on $50 \mathrm{~Hz}$ frequency and it is of 3 phase. Three-phase electric systems have a fourth wire, called "neutral." The"neutral" wire of a three-phase system will conduct electricity if the source and/orthe load are unbalanced. In three-phase systems two sets of voltages and currents can be identified. These are the phase and line voltages and currents. On the other hand, loads can be found connected in star, delta, or a combinationof the two.The concept that a synchronous generator can be used as a motor followed suit. Although Tesla's induction motor replaced the synchronous motor as the choice for the vast majority of electric motor applications, synchronous generators remained the universal machines of choice for the generation of electric power.

The world today is divided between countries generating their power at $50 \mathrm{~Hz}$ and others (e.g., the United States) at $60 \mathrm{~Hz}$. Additional frequencies (e.g., $25 \mathrm{~Hz}$ )can still be found in some locations, but they constitute the rare exception. Large salient-pole rotors are made of laminated poles retaining the windingunder the pole head.

The poles are keyed onto the shaft or spider-and-wheel structure. Salient-pole machines have an additional winding in the rotating member. This winding, made of copper bars short-circuited at both ends, is embedded in the head of the pole, close to the face of the pole. The purpose of this winding is to start the motor or condenser under its own power as an induction motor, and take it unloaded to almost synchronous speed, when the rotor is "pulled in" by the synchronous torque. The winding also serves to damp the oscillations of the rotor around the synchronous speed, and is therefore named the damping-winding (also known as amortisseurs or damper-windings).

\section{Existing System}

The conventional method Supervisory Control And Data Acquisition (SCADA)for plant monitoring may be uneconomical if applied to generator unit in power plant due to high cost, so integrated automation and control has become the solution for making it efficient and cost effective.

- Communication of the system between different computers is not easy, resulting in configuration problems

- Data processing and databases have to be duplicated across all computers in the system, resulting in low efficiencies

- There is no systematic approach to acquiring data from the plant devices - if two operators require the same data, the RTU (Remote Terminal Unit) is interrogated twice.

\section{Proposed System}

LabVIEW stands for Laboratory Virtual Instrumentation Engineering Workbench. As the name indicates, it is a virtual engineering environment with the integration of hardware and software and a full control system, and focuses on the supervisory control level. As such, it is a purely software package that is positioned on top of hardware to which it is interfaced, in general via Programmable Logic Controllers (PLCs), or other commercial hardware modules.

LabVIEW is used to simulate the functional, input and output parameters of the generator uint for the following reasons.

- To reduce the Man Power, so the Labor Cost also be optimized. 
- Analysis of System Response Time(SRT) using NI LabVIEW with the exciting SRT of SCADA software.

- Helpful in Emergency Periods like Unit Synchronization, Unit Shutdown...

A LabVIEW simulation of a laboratory based generator in thermal power plant developed using LabVIEW data logging and supervisory control (DSC) module has capacity to supervise the operation and data acquisition. A simulation has capacity for supervising a plant operation. A simulation has facility to acquire the value of temperature and pressure which are sensed by a temperature sensor and pressure sensor respectively. In this Simulation we can supervise high and low level position of level sensor available at mini thermal power plant setup. Measurement of previous process data can supervise in this simulation in the form of graph plots. Simulation also facilitates alarms for any bad condition or excess condition of an event. Supervisory control of generator in thermal power plant is shown in figure 1. Data acquisition is also possible in this LabVIEW simulation. Data are acquired from the various input, functional and output parameters of generator, Linear bearing temperature sensor, stator water flow sensor, water conductivity sensor, linear bearing and excitation transformer temperature sensors with the help of data acquisition hardware.

A. Input, Functional and Output Parameters of Generator:

1. Input Parameters: Excitation Voltage to rotor and RPM of rotor

2. Functional Parameter: Power Factor

3. Output Parameters:Power,Current,Terminal Voltage

FIGURE 1

SIMULATION OF GENERATOR UNIT USING LABVIEW

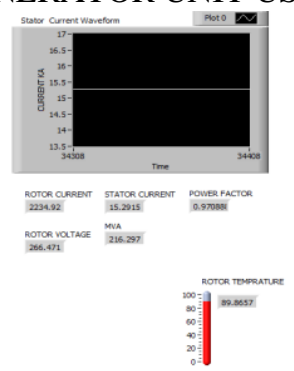

FIGURE 2

BLOCK DIAGRAM IN LabVIEW SIMULATION FOR LABORATORY BASED GENERATOR UNIT IN THERMAL POWER PLANT

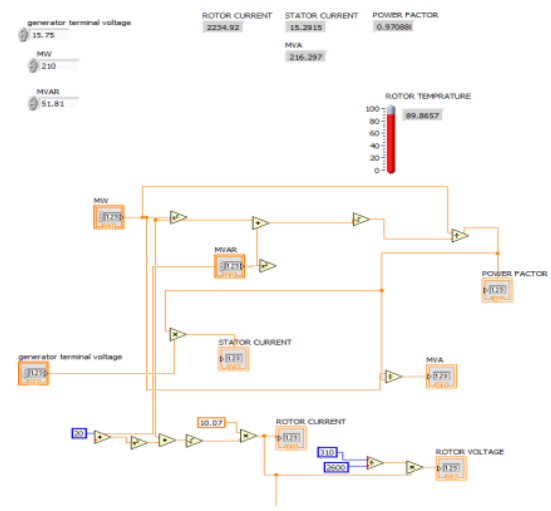

B. Data logging for temperature measurement: Developed LabVIEW simulation has capability to log values of temperature sensed through temperature sensor and acquired by data acquisition system.

C. Alarm Handling: An alarm represents a specific, abnormal condition associated with shared variable or with a user-defined state. You can set and configure alarms for shared variables. An alarm is the alarm information for the specified shared variables. An event is a type of alarm that occurs instantaneously. An event is virtually any instantaneous activity, whereas an alarm typically denotes an abnormal condition, occurs under certain 
specific conditions, or must be acknowledged by the user or configured for automatic acknowledgment. Alarms of an event are acknowledged using this simulation as shown in figure 3 .

FIGURE 3

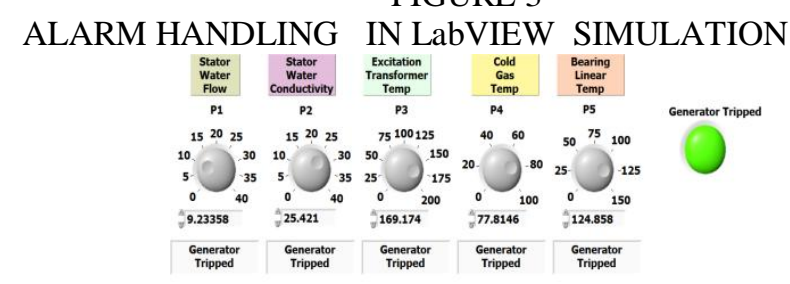

Ss

$$
\begin{gathered}
\text { IV. } \quad \begin{array}{c}
\text { Formula Used } \\
\text { Power Factor }
\end{array}=\frac{\text { Mega Watt }}{\sqrt{\text { MegaWatt }{ }^{2}+\text { MVAR }^{2}}}
\end{gathered}
$$

$M W A=\frac{\text { Mega Watt }}{\text { Power Factor }}$

MVAR $=1.05 \times$ Generator TerminalVoltage $\times 1.732 \times 50$

Rotor Current $=10.07 \times \sqrt{\text { Mega Watt }{ }^{2}+(20+M V A R)^{2}}$

Rotor Voltage $=\left(\frac{310}{2600}\right) \times$ RotorCurrent

$$
\text { Rotor Temperature }=\frac{(115 \times \text { Rotor Current })}{1.1 \times 2600}
$$

\section{Conclusion}

LabVIEW simulation for generator unit is used to supervise mini thermal power plant operation. This simulation has ability for data acquisition from the various temperature sensor, pressure sensor and level sensor. LabVIEW simulation can helps to supervise the position of automatic valves and level sensor. Supervision of Historical data of power plant and alarm indication for an event can possible through this simulation. Data log of temperature and pressure measurement is also possible in this simulation.

\section{References}

[1]. A. Daneels, W.Salter, what is LabVIEW? International Conference on Accelerator and Large Experimental Physics Control Systems, Trieste, Italy 1999

[2]. Baily D, Wright E, Practical LabVIEW for Industry, Elsevier journal of process plants, may 2003.

[3]. Carke G, Rynders D, Wright E, Practical Modern LabVIEW Protocols, Elsevier journal of process plants, 2003

[4]. www.ni.com/labview/labviewdsc/

[5]. www.dewetron.cz/pdf/1msw078_082a.pdf

[6]. M. N. Lakhoua, LABVIEW applications in thermal power plants, International Journal of the Physical Sciences Vol. 5(6), pp. 11751182, June 2010 